\title{
Dynamische Aspekte der Argumentinterpretation: Eine neurokognitive Perspektive
}

\begin{abstract}
Der vorliegende Aufsatz beschäftigt sich mit der Frage, wie Argumente während des Sprachverstehens erkannt werden, welche Eigenschaften einem Argument in Abwesenheit des Verbs zugeschrieben werden und welche Art von Vorhersagen mit der Argumentinterpretation verbunden sind. Ausgehend von der Annahme, dass beim Sprachverstehen in Echtzeit jedes Wort so maximal wie möglich interpretiert wird, werden wir argumentieren, dass die zugrunde liegenden, sprachübergreifend zu findenden Mechanismen durch die Interaktion von typologisch motivierten Prominenzskalen (z.B. Belebtheitshierarchie) beschrieben werden sollten. Diese gestatten nicht nur eine Erklärung bestehender Befunde, sondern besitzen das Potenzial, zentrale Aspekte der Sprachverstehensarchitektur modelltheoretisch abzuleiten. Experimentell liegt der Fokus des Aufsatzes auf der Erfassung elektrophysiologischer-neuronaler Aktivierungsmuster, da diese uns im Gegensatz zu Urteilen oder Korpusverteilungen einen unmittelbaren Einblick in die Verarbeitung im Echtzeitbereich gestatten.
\end{abstract}

\section{Einleitung}

Betrachtet man Sprache aus einer Echtzeitperspektive, so wird schnell deutlich, dass in vielen Sprachen der Welt Nomen und Präpositionalphrasen interpretiert werden müssen, ohne dass man weiß, welche Rolle diese in der später durch das Verb festzulegenden Handlung besitzen. Nun könnte man der dadurch entstehenden Unsicherheit entgehen, wenn man diese Nomen und Präpositionalphrasen nicht sofort versucht hinsichtlich ihrer Funktion zu interpretieren, sondern einfach bis zum Auftreten des Verbs wartet. Doch so arbeitet unser Sprachverstehenssystem genau nicht. Durch den unmittelbaren Versuch der maximalen Interpretation bei gleichzeitiger Inkaufnahme einer möglichen späteren Revision vermeiden wir, dass die präverbale Information sich unstrukturiert möglicherweise so sehr ansammelt, dass wir Teile davon einfach vergessen, wenn der Moment kommt, an dem das den Rahmen aufspannende Verb auftritt (für die Idee, dass unmittelbare maximale Strukturierung Arbeitsgedächtniskosten reduziert, siehe Frazier/Fodor 1978). Doch was bedeutet maximale Interpretation? Welche Art von Merkmalen aus dem Lautstrom oder aus einem geschriebenen Satzfragment nutzen wir für die Festlegung, ob es sich um ein mögliches Verbargument handelt und welche Rolle es im zu erwartenden Verbrahmen einnimmt? Der vorliegende 
Beitrag versucht, mit Hilfe ausgewählter Daten aus der neurokognitiven Sprachforschung darauf Antworten zu geben. Er wird darüber hinaus zeigen, wie sprachübergreifend ähnliche Mechanismen genutzt werden, um Argumente hinsichtlich ihrer Funktion zu determinieren und von anderen potenziellen Argumenten möglichst eindeutig abzugrenzen.

\section{Inkrementalität: Phänomen und Konsequenzen}

Beginnen wir mit dem eigentlichen Grundproblem. Bis auf wenige Ausnahmen (z.B. Pritchetts, ,head driven processing account" $(1988,1992))$ ist man seit den Anfängen der psycholinguistischen Forschung stets davon ausgegangen, dass während des Sprachverstehens die Bedeutungskonstitution sich kontinuierlich, d.h. zyklisch und maximal an jedem aus dem Lautstrom identifizierten Segment, entfaltet und dabei - was wesentlich ist - unabhängig vom Verb ablaufen kann. Dieses als „Inkrementalität“ bekannte Phänomen konnte durch zahlreiche Studien empirisch untermauert werden (siehe insbesondere Crockers „principle of incremental comprehension“ 1994, aber auch Marslen-Wilson 1973). Man kann es sich anhand eines sehr einfachen Beispiels verdeutlichen. Wenn das Satzfragment Alle börten, dass der König von Thailand ... gelesen oder gehört wird, dann gibt es mit der Verarbeitung der Präpositionalphrase von Thailand bereits bestimmte Annahmen über die syntaktische Struktur, die dann sichtbar werden, wenn mit dem nächsten Wort erfuhr der Satz beendet wird. Muttersprachler des Deutschen sind beim Auftreten des Verbs in der Regel überrascht und haben für einen kurzen Moment den Eindruck, dass der Satz nicht interpretierbar ist. Erst durch eine Reanalyse, d.h. durch die Uminterpretation der Präpositionalphrase zum Argument des Verbs erfahren, kommt die korrekte Interpretation zustande. Die Notwendigkeit zur Reanalyse zeigt aber auch, dass wir bereits zum Zeitpunkt der Verarbeitung der Präposition (bzw. der Präpositionalphrase) eine Entscheidung zugunsten der das Nomen modifizierenden Lesart getroffen haben müssen, die es nun zu korrigieren gilt (für experimentelle Befunde und ihre theoretische Einordnung siehe Konieczny et al. 1997).

Mit dem nächsten Beispiel soll hier - wiederum rein deskriptiv - eine weitere Eigenschaft des Sprachverstehens vorgestellt werden. Dabei geht es nun bereits um eine Frage der Argumentinterpretation und ihrer Tiefe. Hören oder lesen deutsche Muttersprachler den Satz Alle wussten, dass Martin Lehrerinnen widersprechen, so gibt es auch hier wieder einen kurzen Moment einer für Holzwegsätze dieser Art oft zu beobachtende Überraschung, die messbar zu einem Absinken der Akzeptabilität führt bzw. in Veränderungen in neurophysiologischen/-anatomischen Mustern resultiert (siehe Haupt et al. 2008 für einen aktuellen Überblick). Diese entsteht, wenn das Verb nicht wie erwartet im Singular auftritt, sondern durch die Pluralmarkierung eine 
Objekt-vor-Subjekt-Abfolge (OS) erzwingt. Der Effekt, der übrigens auch zu beobachten ist, wenn man den Satz in einen Kontext einbettet, der die OS-Lesart stützt, ${ }^{1}$ basiert darauf, dass bei der Verarbeitung von Martin diesem ambigen Argument lokal die grammatische Rolle des möglicherweise einzigen syntaktischen Argumentes zugewiesen wird (siehe Bornkessel-Schlesewsky/Schlesewsky 2009), welches den Annahmen der deutschen Syntax folgend damit die grammatische Funktion des Subjekts zugeschrieben bekommt. ${ }^{2}$ Eine Reanalyse wird aber dann notwendig, wenn das Verb nicht

1 Gelegentlich hört man das Argument, dass derartige OS-Abfolgen im Deutschen ja eigentlich nicht gebräuchlich sind und in Isolation wohl niemals zu beobachten sein werden. Ein Beispiel aus dem „Fränkischen Tag“, einer Tageszeitung Oberfrankens, zeigt das Gegenteil zu dieser Annahme. Dort fand sich die folgende Titelzeile: Trotz. Frost sollten Rehe Wanderer nicht füttern. Weitere Belege lassen sich ohne Probleme auch in anderen Registern finden.

2 In Schlesewsky/Bornkessel (2004) haben wir basierend auf einem Vergleich zwischen kasusambigen und eindeutig kasusmarkierten Argumenten im Deutschen argumentiert, dass die inkrementelle Interpretation kasusambiger Nominalphrasen tatsächlich nur bis zur Zuweisung der grammatischen Funktion geht. Von dort wird nicht auf die thematische Rolle, wie Agens, geschlossen. Das bedeutet nicht, dass thematische Rollenzuweisungen keine Bedeutung besitzen, doch lassen sich Effekte, die sich auf diese zurückführen lassen, nur dann beobachten, wenn eine eindeutige Kasusmarkierung oder Verbinformationen vorliegen. Hinsichtlich der Verbinformation lässt sich aber auch zeigen, dass deren thematische Eigenschaften im Kontext der Verarbeitung kasusambiger Argumente lokal vernachlässigt werden können, wenn sie mit der Zuweisung der grammatischen Funktion konfligieren. In Bornkessel/Schlesewsky (2006a) berichten wir einen Reanalyseeffekt auf der nominativmarkierten NP in Sätzen mit dem finiten Verb an zweiter Position, wie Wabrscheinlich gefällt Maria der Mann ... Unter der Annahme, dass das Verb gefallen seine höchste thematische Rolle an das erste mögliche Argument vergibt, sollte diesem Argument die grammatische Funktion des Objektes zugewiesen werden. Der Reanalyseeffekt zeigt aber, dass das offenbar nicht geschieht.

Basierend auf diesen Arbeiten haben wir auch dafür argumentiert, dass es während des inkrementellen Sprachverstehens keine automatische Kasuszuweisung an ein kasusambiges Argument gibt, d.h. ein Argument kann zwar die grammatische Funktion des Subjektes zugewiesen bekommen und damit die Erwartung für ein bestimmtes Kongruenzmerkmal auf dem Verb auslösen, diese Zuweisung der grammatischen Funktion löst aber keine Zuweisung eines Kasusmerkmals aus. Dieser experimentell motivierte Unterschied zwischen Kasus und grammatischer Funktion wird im Folgenden für die Argumentation zentral sein. Ebenfalls sei an dieser Stelle angemerkt, dass wir bereits in Bornkessel/Schlesewsky/Friederici (2003), aber insbesondere in Schlesewsky/Bornkessel (2004) und in Bornkessel/Schlesewsky (2006) argumentiert haben, dass es mit dem Konzept der generalisierten semantischen (thematischen) Rolle im Sinne von Primus (1999) und van Valin (Foley/van Valin 1984) zumindest aus der psycho-/neurolinguistischen Perspektive eine dritte Dimension gibt, die sich nicht automatisch aus den beiden anderen hier genannten Dimensionen (Kasus und grammatische Funktion) ableiten lässt. Generalisierte semantische Rollen erlauben aus der experimentellen Perspektive Vorhersagen und Erklärungen für die zu beobachtenden Effekte, die sich mit den anderen zuvor genannten Dimensionen und mit klassischen thematischen Rollenkonzeptionen (z.B. Jackendoff 1972; Grimshaw 1990) nicht ableiten lassen. Während wir in der ursprünglichen Motivation (Bornkessel et al. 2003) auf der Grundlage der Protorollenkonzeption von Dowty (1991) und Primus (1999) argumentierten, erscheint seit unseren Arbeiten ab 2006 zu diesem Thema ein Modell, welches auf der Actor/UndergoerKonzeption von van Valin beruht und den Dependenzansatz zwischen diesen Rollen im Sinne von Primus (1999) in die Relation der generalisierten semantischen Rollen integriert (Bornkessel/Schlesewsky 2006a, b). 
mit diesem Nomen kongruiert. Dass die Notwendigkeit zur Korrektur nicht nur messbar, sondern auch bewusst nachvollziehbar ist, zeigt der kognitive Aufwand, den Sprecher des Deutschen benötigen, um zur richtigen Interpretation zu gelangen (siehe Sturt/Crocker 1996 für ein Modell zur Effektstärke bei syntaktischen Reanalysen). Bemerkenswert ist in diesem Kontext aber auch, dass die Subjektspräferenz (Frazier/Flores d'Arcais 1989; Schriefers/Friederici/Kühn 1995), ${ }^{3}$ d.h. die Zuweisung der Subjektsfunktion an das erste mögliche Argument, mitunter so schnell (und einfach) reanalysiert werden kann, dass man den Aufwand nicht mehr bewusst „erlebt“. Akzeptabilitätsstudien zeigen, dass im Gegensatz zu dem zuvor genannten Beispiel der Satz Alle wussten, dass Martin Lehrerinnen gefallen signifikant besser bewertet wird (Schlesewsky/Bornkessel 2003; Bornkessel et al. 2004). Das ist einerseits überraschend, da auch hier - sollten die Prozesse der Zuweisung grammatischer Funktionen genauso automatisch ablaufen - die SO-Abfolge auf dem Verb revidiert werden muss. Andererseits ist die höhere Akzeptabilität sofort einsichtig, da das so genannte Objektexperiencerverb gefallen bei der Reanalyse durch seine inverse thematische Rollenzuweisung, d.h. die hierarchisch höhere thematische Rolle wird dem syntaktisch hierarchisch niedrigeren Argument zugewiesen und vice versa (Primus 1999; Wunderlich 1997; Fanselow 2000), den Weg zur korrekten OS-Interpretation weist. Dieser Prozess läuft nun aber so schnell ab, dass wir den kognitiven Nachteil, der durch die Reanalyse ausgelöst wird, in dem Moment, an dem uns die Bedeutung des Satzes bewusst wird, nicht mehr wie im Kontext der oben beschriebenen Sätze nachempfinden können.

An dieser Stelle entfalten nun Messmethoden ihren Vorteil, die neurophysiologisch oder neuroanatomisch Aktivierungsveränderungen direkt am oder im Kortex messen. Insbesondere Messungen mit Hilfe des Elektroenzephalogramms (EEG) sollen im Folgenden im Vordergrund stehen. Diese haben den Vorteil, dass sie im Bereich von Millisekunden Aktivierungsveränderungen erfassen können. ${ }^{4}$ EEG-Messungen bestätigen nun, dass sich

Für das Deutsche wurde die Präferenz, ambige initiale Nominalphrasen als Subjekt zu interpretieren, erstmals systematisch in Hemforth (1993) für Deklarativsätze und in Farke (1994) für $w h$-Strukturen untersucht.

4 Im Jahre 1929 veröffentlichte der Jenaer Neurologe Hans Berger eine Arbeit, in der er zeigte, dass sich an der menschlichen Schädeloberfläche elektrische Hirnströme ableiten lassen, die durch kognitive Aufgaben, wie das Lösen einer arithmetischen Aufgabe, moduliert werden können (Berger 1929). Diese Veränderungen der elektrischen Aktivität lassen sich mit Hilfe so genannter ereigniskorrelierter Potenziale (EKPs) untersuchen und charakterisieren. EKPs sind hirnelektrische Potenzialverschiebungen, die in einem zeitlich exakt fixierbaren Zusammenhang zu einem sensorischen, motorischen oder kognitiven Ereignis stehen. Die Messung von EKPs erfolgt durch die Ableitung von Synapsenpotenzialen an der Schädeloberfläche. Der Tradition folgend, wurden die ersten Sprachuntersuchungen mittels EKPs auf die Analyse von Unterschieden zwischen den Hemisphären beschränkt. Obwohl diese Arbeiten die Linkslateralisierung von Sprache, wie sie aus Patientenstudien bereits bekannt war, bestätigten, erbrachten sie keine wesentlichen Fortschritte im Hinblick auf ein erweitertes Ver- 
die beiden genannten Beispiele hinsichtlich des auftretenden Konfliktes vollkommen gleich verhalten, da sie die identische neurophysiologische Reaktion zeigen (Bornkessel et al. 2004). Die dort zu beobachtende Negativierung zwischen 300 und 500 ms wird als N400 bezeichnet und reflektiert die Notwendigkeit der Korrektur der grammatischen Funktion der beiden zuvor verarbeiteten Argumente. ${ }^{5}$ Es zeigt sich also, dass in beiden Sätzen das Auftreten eines Verbs mit einer unerwarteten Numerusflexion zu erhöhten neuronalen Aktivierungen führt und daher die Annahme abzuleiten gestattet, dass der eigentliche Konflikt identisch ist.

Abbildung 1 zeigt die neurophysiologischen Veränderungen, die man beobachten kann, wenn - wie hier - bei der Verarbeitung des Verbs (y-Achse signalisiert den zeitlichen Beginn der Wortpräsentation) die notwendige Korrektur zur OS-Abfolge deutlich wird. Sichtbar ist, dass sich beide Verbtypen

ständnis sprachlicher Prozesse. Erst mit den Arbeiten von Kutas und Hillyard (1980) wurde ein Weg beschritten, der einen direkten Zugang zu den hier beschriebenen sprachlichen Phänomenen erlaubt. Da die „natürliche“, zufällig entstehende Grundaktivität des Gehirns $(50-150 \mu \mathrm{V})$ um ein Vielfaches größer ist als die durch den kognitiven Reiz ausgelöste Aktivierung $(1-8 \mu \mathrm{V})$, werden die kritischen Reize mehrfach wiederholt und anschließend gemittelt. Durch dieses Verfahren wird einerseits die Grundaktivität eliminiert, während andererseits gleichzeitig die reizrelevante Aktivität isoliert werden kann. Die aus der Mittelung resultierenden, digitalisierten Daten lassen sich als Kurven visuell darstellen. Die in Sprachverstehensexperimenten zu beobachtenden Effekte (Komponenten) werden aus einer funktionalen Perspektive interpretiert, d.h. es werden stets zwei Bedingungen miteinander verglichen, die nur einen minimalen, genau definierten und theoretisch motivierten Unterschied besitzen. Die aus dem Vergleich resultierenden Differenzen werden auf der Basis ihrer Latenz (die Zeitspanne zwischen der Darbietung des Reizes und dem Auftreten des Effektes), Topografie (an welchen Elektrodenpositionen der Effekt auftritt, siehe Abbildung 6), Polarität (die Richtung der Auslenkung gegenüber der Kontrollbedingung, d.h. positiv oder negativ) und Amplitude (der Grad der maximalen Ausprägung) klassifiziert. Im Laufe der vergangenen Jahre ist es gelungen, eine Reihe von sprachrelevanten Komponenten zu identifizieren, die Aussagen über die Art der sprachlichen Architektur und ihren sprachübergreifenden Charakter gestatten (für einen Überblick siehe Bornkessel-Schlesewsky/Schlesewsky 2009a).

$5 \quad$ An dieser Stelle scheint eine Anmerkung zu den hier vorgestellten Komponenten notwendig. Während man bis zur Mitte der 90er Jahre des vergangenen Jahrhunderts - und basierend auf ersten Befunden vor allem aus dem Englischen - hoffte, mit Hilfe von EEG-Komponenten zwischen sprachlichen Domänen unterscheiden zu können (Garnsey 1993), geht man in der aktuellen Forschung davon aus, dass ein solcher Schluss nicht mehr aufrechterhaltbar ist (z.B. Urbach/Kutas 2010). So ist die Annahme, dass man durch das Auftreten einer N400 auf lexikalische oder semantische Prozesse schließen könnte, während das Vorkommen einer P600 (Positivierung mit einem Maximum um 600 ms) syntaktische Prozesse reflektiert, empirisch momentan nicht mehr zu rechtfertigen (siehe Bornkessel-Schlesewsky/Schlesewsky 2009a, Kapitel 16 für eine zusammenfassende Darstellung des Problems). Beispiele, die dieses Problem illustrieren, sind die so genannte ,semantische P600“ (At breakfast, the eggs would eat, Kuperberg et al. 2003; Kolk et al. 2003); Positivierungen bei Verletzung von Antonymrelationen (Das Gegenteil von schwarz ist gelb, Roehm et al. 2007) bzw. bei niedrigfrequenten Nomen am Satzanfang (Welche Trappistin/Lebrerin ..., Bergmann 2010) oder N400Effekte bei morphosyntaktischen (split-Ergativitäts-)Verletzungen im Hindi (Choudhary et al. 2009) oder bei regelbasierten phonologischen Prozessen (Janssen et al. 2006). 
gleich verhalten. Man beachte, dass die Kurven niemals absolut, d.h. im Koordinatensystem interpretiert werden, sondern nur relativ zueinander (siehe Fußnote 4).

A. Aktiv-Verben

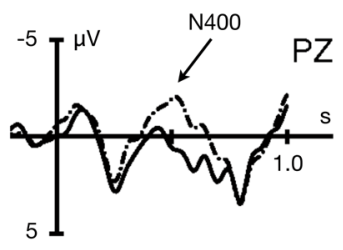

\section{B. Objektexperiencer-Verben}
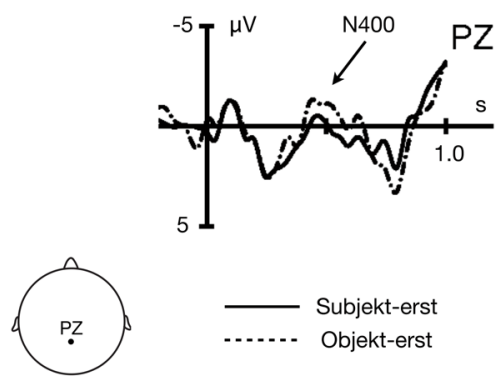

Subjekt-erst

Objekt-erst

Abb. 1: Verarbeitung von lokal ambigen NP-NP-V-Strukturen (Abbildung adaptiert aus Bornkessel et al. 2004)

Dass sich neurophysiologisch die beiden Verbtypen in ihrem Reanalyseverhalten nicht unterscheiden, dass aber behaviorale Unterschiede im Antwortverhalten zu finden sind, bestätigt die zuvor bereits beschriebene Annahme, dass es nicht der Konflikt selbst, d.h. die Erwartungsverletzung, ein singular markiertes Verb zu finden, sondern die Korrektur der Zuweisung der grammatischen Funktion ist, die die Akzeptabilitätsunterschiede verursacht (Bornkessel et al. 2004).

Lässt man die bis zu diesem Zeitpunkt angeführten Argumente Revue passieren, so kann man festhalten, dass das menschliche Sprachverarbeitungssystem a) inkrementell, d.h. kontinuierlich, die zu jedem Zeitpunkt angebotenen Informationen verarbeitet, b) im Falle von Mehrdeutigkeiten (Ambiguitäten) eine Lesart präferiert, c) aus der Lesart Erwartungen über nachfolgende Informationen (z.B. Numerus des Verbs) ableitet, und d) die Fähigkeit zur Korrektur (Reanalyse) besitzt, die unterschiedlich schwer sein kann und somit das Spektrum von unbewusster Reanalyse bis hin zur Nichtinterpretierbarkeit aufspannt (siehe die zuvor zitierten Referenzen und Bornkessel-Schlesewsky/Schlesewsky (2009a, Kapitel 7) für eine aktuelle Darstellung). Alle diese Eigenschaften bilden die Grundlage der im Folgenden zu beschreibenden Phänomene.

\section{Argumente im Wettbewerb}

Um zu verstehen, was „Argumente im Wettbewerb“ wirklich bedeutet, stellen wir uns zunächst einmal die folgende Situation aus dem wirklichen Leben vor, die viele von uns einfach nachvollziehen können. Nehmen wir 
also an, dass an einer Universität und in einer philologischen Fakultät eine Professur für Sprachwissenschaft ausgeschrieben wurde. Ein erster Aspekt, der die Besetzung dieser Stelle wesentlich beeinflusst, ist die Berufungskommission und deren Zusammensetzung. Selbst unter der Annahme, dass diese Kommission alle ethischen und moralischen Grundsätze bei einer solchen Arbeit wahrt, hat sie trotzdem bestimmte Präferenzen bzw. befindet sich im Spannungsfeld verschiedener Präferenzen der Kommissionsmitglieder. Ebenso wichtig ist der Aspekt des Bewerberfeldes. Hier gibt es zwei Seiten. Sollte es - und hier weichen wir mal ein wenig von der Realität ab nur einen Bewerber oder eine Bewerberin geben, dann sind dessen/deren wissenschaftlichen Eignungen und Präferenzen zwar nicht unwichtig, sie entfalten ihre eigentliche Bedeutung aber erst in der Konkurrenz. Solange man der alleinige Vertreter/Bewerber ist, kann man sich schon Schwächen leisten, denn objektive Zwänge (z.B. die Sicherstellung der Lehre und der Prüfungen) lassen eine gewisse Toleranz zu. In dem Moment - hier sind wir wieder in der Realität -, bei dem das Bewerberfeld aus mehreren Personen besteht, erlangen die Merkmale jedes einzelnen Bewerbers durch die kompetitive Situation eine neue Qualität. Jetzt geht es nicht mehr darum, was man selbst ist, sondern in welcher Relation, d.h. mit welchen distinktiven Eigenschaften, man zu dem oder den anderen steht. Eine einfache Entscheidung ist nur dann möglich, wenn die Bewerber klar voneinander unterscheidbar sind. Ähneln sie sich zu sehr, dann wird die Entscheidung nicht nur schwieriger, sondern der Ausgang des Verfahrens besitzt eine höhere Wahrscheinlichkeit zur Zufälligkeit.

Wenn sich nun der geneigte Leser etwas irritiert fragt, was dieses Beispiel mit Sprachverstehen und Argumentverarbeitung aus einer dynamischen Perspektive zu tun hat, dann können die Autoren ihm/ihr versichern, dass er/sie soeben die Grundeigenschaft der Argumentinterpretation und der Verarbeitung dieser kennen gelernt hat. Denn so unwahrscheinlich, wie es auf den ersten Blick erscheinen mag: Sprachübergreifende Studien (siehe die Referenzen zu den Abbildungen 3-5) deuten darauf hin, dass wir während des Sprachverstehens exakt so vorgehen, wie in der oben geschilderten Situation (Bornkessel-Schlesewsky/Schlesewsky 2009b). Dabei reflektieren Verben die Eigenschaften und Anforderungen der jeweiligen Berufungskommission und die Argumente sind die Kandidaten, die um den ersten Platz auf der zu erstellenden Liste konkurrieren. Vernachlässigen wir der Einfachheit halber zuerst einmal das Verb, da es uns nicht um die spezifischen Anforderungen des Stellenprofils geht, sondern nur um die Tatsache, dass am Ende eine Liste erstellt wird, die einen Erstplatzierten besitzt. Damit besitzen für die folgenden Ausführungen die Bewerber die zentrale Rolle. Wir beschränken uns zunächst auf das Deutsche und illustrieren hier alle wesentlichen Aspekte. In einem weiteren Schritt (Abschnitt 4) werden wir dann zeigen, dass sich diese auch sprachübergreifend beobachten lassen. 
Betrachten wir also zunächst einmal aus einer inkrementellen Perspektive die Verarbeitung eines satzinitialen kasusmarkierten Argumentes (welcher Jäger) in dem Satzfragment Jane fragte sich, welcher Jäger... Welche Annahmen resultieren aus der Verarbeitung des nominativmarkierten Argumentes? Auf dem aktuellen Argument lässt sich selbst noch nicht viel beobachten, überraschenderweise auch dann nicht, wenn das Nomen unbelebt ist und einen eher suboptimalen Handlungsverursacher darstellt. Dies steht im Gegensatz zu Beobachtungen aus dem Englischen, da bereits an dieser Stelle suboptimale, d.h. unbelebte Subjekte zu Verarbeitungskosten führen (Weckerly/Kutas 1999, siehe Fußnote 10). Erweitert man aber den Satz durch ein zweites Argument (Jane fragte sich, welcher Jäger den Gärtner ...) zu einer transitiven Lesart, so lässt sich im EEG auf dem zweiten Argument erhöhte Aktivierung messen, die man als Kosten der Erweiterung von einer ursprünglich intransitiven auf eine transitive Lesart interpretieren kann (Bornkessel/Fiebach/Friederici 2004).

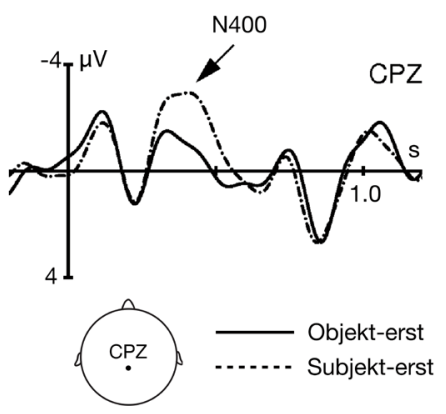

Abb. 2: Negativierung für die Verarbeitung eines zweiten (nicht vorhersagbaren) Argumentes im Vergleich zu einem zweiten Argument, welches durch einen initialen Akkusativ (Jane fragte sich, welchen Jäger ...) erwartbar ist (Abbildung adaptiert aus Bornkessel/Fiebach/Friederici 2004). Man beachte, dass die Kurven niemals absolut, d.h. im Koordinatensystem interpretiert werden, sondern nur relativ zueinander (siehe Fußnote 4).

Bei Abbildung 2 zeigt sich also eine erste Präferenz, die an das oben genannte Bewerbungsbeispiel anschließt und auf die wir bereits im Kontext der Verarbeitung kasusambiger Argumente kurz hingewiesen haben. Ohne explizite Evidenz wird auch ein initialer Nominativ so verarbeitet, als ob er das einzige Argument eines (somit intransitiven) Ereignisses oder Zustandes darstellt. Dabei ist es lokal unerheblich, ob er viele prototypische Merkmale (z.B. die genannte Belebtheitseigenschaft) besitzt oder sich eher suboptimal hinsichtlich dieser Anforderungen verhält. Wichtig ist einzig, dass weder weitere Argumente angenommen noch spezifische Anforderungen an Verbeigenschaften gestellt werden. Es wird zu diesem Zeitpunkt nur angenommen, dass der Nominativ das einzige Argument des Ereignisses ist und dass das obligatorisch zu erwartende Verb nur ein syntaktisch zu realisie- 
rendes Argument benötigt. ${ }^{6}$ Muss die Annahme der „Einzigartigkeit“ revidiert werden, geht das mit messbaren Kosten einher (Bornkessel-Schlesewsky/Schlesewsky 2009b). Einerseits führt die Erweiterung von einer intransitiven zu einer transitiven Relation zu Erweiterungskosten (siehe Abschnitt 4 sowie die Diskussion zu Abbildung 4). Momentan wichtiger ist aber, dass durch das Auftreten eines zweiten Handlungsteilnehmers die lexikalischen Eigenschaften des Nominativs plötzlich bedeutsam werden. Dieses zeigt sich in der im Folgenden dargestellten Studie, in der es um die Vorhersage eines noch zu verarbeitenden Nominativarguments geht. Diese kann durch ein initiales Akkusativargument erzeugt werden, da das Objekt i.d.R. zur Erstellung einer syntaktisch korrekten Form nicht nur ein Verb sondern auch ein Nominativargument erfordert. Obwohl - wie oben kurz erwähnt - ein initialer unbelebter Nominativ keine Verarbeitungsnachteile zeigt, lassen sich auf diesem dann erhöhte Aktivierungen beobachten, wenn er wie in Jane fragte sich, welchen Gärtner der Zweig streifte einem zuvor verarbeiteten Akkusativ folgt. Dies illustriert Abbildung 3 aus einer Studie von Roehm und Kollegen aus dem Jahre 2004.

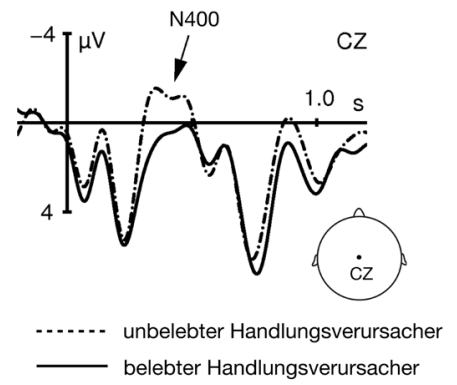

Abb. 3: Negativierung für die Verarbeitung eines unbelebten, durch einen vorangehenden Akkusativ vorhergesagten Nominativarguments im Vergleich zu einem belebten Nominativ (der Zweig im Vergleich zu der Jäger), gemessen auf dem kritischen Argument (Abbildung adaptiert aus Roehm et al. 2004). Man beachte, dass die Kurven niemals absolut, d.h. im Koordinatensystem interpretiert werden, sondern nur relativ zueinander (siehe Fußnote 4).

Wichtig ist es in diesem Zusammenhang zu erkennen, dass es weniger die Kombination von Nominativ und Belebtheit ist, die diesen Effekt verursacht, sondern die durch die Verarbeitung eines Akkusativarguments ausgelöste Erwartung für die Eigenschaften dieses Nominativarguments. Aus der Perspektive des Objektes und als Konsequenz der inkrementellen Interpre-

Das hier verwendete Konzept der Transitivität basiert auf den Arbeiten von DeLancey (1981), Comrie (1989) und de Swart (2007, für die Hörerperspektive). Transitivität ist demnach nicht nur eine dem Verb inhärente Eigenschaft, die syntaktisch und/oder semantisch die Zahl der Handlungsteilnehmer festlegt, sondern bestimmt sich auch durch die Eigenschaften der Handlungsteilnehmer selbst (siehe auch Hopper/Thompson 1980 für einen ähnlichen Ansatz). 
tation soll dieses nun nicht nur die entsprechende morphologische Markierung haben, sondern es soll auch die Eigenschaft eines optimalen Handlungsverursachers besitzen, nämlich belebt sein. Wird diese Eigenschaft nicht erfüllt, zeigt die erhöhte Aktivierung die Erwartungsverletzung und damit einen Verarbeitungsnachteil. ${ }^{7}$

An dieser Stelle wollen wir einmal kurz über die EEG-Messungen hinausgehen und uns einen Befund aus einer Studie anschauen, bei dem mit Hilfe bildgebender Verfahren (funktionelle Magnetresonanztomographie, fMRT) Aktivierungsänderungen sichtbar gemacht werden können. In Grewe et al. (2007) wurden transitive Sätze verglichen, bei denen das Akkusativargument entweder als belebt (Der Pirat hat die Prinzessin geraubt.) oder als unbelebt (Der Pirat hat den Schatz geraubt.) realisiert wurde. Eine bestimmte anatomische Region (der linke Sulcus temporalis superior, siehe Abbildung 6) zeigt im direkten Vergleich der beiden Satzrealisierungen erhöhte Aktivierung, wenn beide Handlungsteilnehmer belebt sind. Die Autoren interpretieren diese Aktivierung ganz im Sinne des hier dargestellten Wettbewerbsprinzips. Werden die Argumente in den Verbrahmen eingefügt, konkurrieren sie um die Position des höchsten Argumentes (des Handlungsverursachers). Je ähnlicher sich die Argumente in ihren Eigenschaften sind, desto höher ist der Aufwand der Zuweisung der Argumente zu den Leerstellen. Dieser Aufwand ist, wenn man den Autoren folgen möchte, kostenträchtig und messbar. Einfach ist die Verarbeitung also dann, wenn beide sich in allen relevanten Dimensionen - welche das sind, werden wir gleich noch näher betrachten - klar voneinander unterscheiden. ${ }^{8}$ Dabei deutet die Studie darauf hin, dass neben einem eindeutigen morphologischen Merkmal (Kasus) auch Belebtheit eine wichtige Rolle im Verarbeitungsverhalten zukommt. Wie bereits kurz angedeutet, kann man neben den beiden Eigenschaften noch weitere identifizieren, die die sprachliche Informationsverarbeitung vereinfachen. In Bornkessel-Schlesewsky/Schlesewsky (2009b) haben wir basierend auf einer Reihe von Sprachverstehensdaten, auf die hier aufgrund von Platzbeschränkungen nicht näher eingegangen werden soll, die folgende Hypothese aufgestellt:

\footnotetext{
Daten aus dem Chinesischen stützen die Annahme, dass die zu beobachtenden Effekte auf die Erwartungsverletzung zurückgehen und nicht auf die Merkmalskombination (Philipp et al. 2008). Da ein Subjekt - unter der Annahme, dass das Chinesische die grammatische Funktion Subjekt besitzt - bzw. ein Handlungsverursacher in Abwesenheit expliziter Verbinformationen keine Vorhersage eines zweiten Argumentes gestattet, sollte die Verarbeitung eines Objektes bzw. Handlungserleidenden lokal keine Unterschiede hinsichtlich einer Belebtheitsvariation zeigen. Tatsächlich gibt es keine messbaren Kosten im EEG.

8 Diese Idee der wettbewerbsbasierten Konkurrenz findet sich in der psycholinguistischen Literatur an mehreren Stellen und wird i.d.R. unter interferenzbasierten Ansätzen zusammengefasst (z.B. Vosse/Kempen 2000; Gordon et al. 2001; Lewis/Vasishth 2005; van Dyke/ McElree 2006).
} 
The interface hypothesis of incremental argument interpretation:

Incremental argument interpretation (i.e. role identification and assessment of role prototypicality) is accomplished by the syntax-semantics interface, that is, with reference to a cross-linguistically defined set of prominence scales and their languagespecific weighting.

The relevant prominence scales are:

a. morphological case marking (nominative $>$ accusative / ergative $>$ nominative)

b. argument order (argument $1>$ argument 2$)^{9}$

c. animacy (+animate $>-$ animate)

d. definiteness/specificity ( + definite/specific $>-$ definite/specific)

e. $\operatorname{person}(1 \mathrm{st} / 2 \mathrm{nd}$ person $>3 \mathrm{rd}$ person)

(Bornkessel-Schlesewsky/Schlesewsky 2009b, S. 28)

Dem sich in der typologischen Literatur heimisch fühlenden Leser wird natürlich sofort auffallen, dass das Konzept der Prominenzskalen hier nicht neu eingeführt wurde, sondern, dass es zu diesem Thema bereits zahlreiche Arbeiten gibt (z.B. Silverstein 1976; Comrie 1989; Aissen 2003; Croft 2003 für eine Auswahl). Darüber hinaus gibt es mit DeLancey (1981) bereits aus der Typologie hervorgehende Annahmen, dass Informationsverarbeitung dann einfach ist, wenn die Argumente in einer unmarkierten transitiven Relation zueinander stehen, d.h. auf möglichst vielen dieser oben genannten Skalen eindeutig distinkt sind. Spannend ist nun, dass es immer mehr Daten aus dem Bereich der Psycho- und Neurolinguistik gibt, die darauf hin deuten, dass den Prominenzskalen bei der Argumentinterpretation nicht nur eine erhebliche Bedeutung zukommt, sondern dass diese auch das Potenzial zu besitzen scheinen, die Architektur des menschlichen Sprachverstehenssystems bzw. die der menschlichen Sprache überhaupt in bedeutendem Maße zu formen. Doch davon mehr im folgenden Abschnitt.

In der Literatur zur Abfolge der Argumente im deutschen Mittelfeld haben unter anderem Lenerz (1977), Uszkoreit (1986) und Müller (1999) darauf hingewiesen, dass neben der grammatischen Funktion auch Belebtheit, Pronominalität etc. sequenzrelevant sind. Mit dem vorliegenden Modell wollen wir diese Ansätze erweitern. Die genannten Merkmale sind nicht nur relevant für die Abfolge, sondern für die Verarbeitung sprachlicher Informationen generell. Neurolinguistisch lässt sich diese Position wunderbar illustrieren. Die Verletzung der z.B. durch Lenerz (1977) formulierten Abfolgeregeln führt zu Aktivierungsveränderungen im Pars opercularis des linken Gyrus frontalis inferior, der sich im linken Frontallappen befindet und der Teil des bekannten Broca-Areals ist. So zeigt nicht nur die Verletzung der Regel Nom > Akk (,, " bedeutet steht linear vor) Aktivierungsmodulationen (Friederici et al. 2006; Röder et al. 2002), sondern auch Abfolgeprinzipien, wie Pronomen > nichtpronominale NPs (Grewe et al. 2005), höhere thematische Rolle > niedrigere thematische Rolle (Bornkessel et al. 2005), belebt > unbelebt (Grewe et al. 2006), definit/spezifisch > indefinit/unspezifisch (Bornkessel et al. 2009) korrelieren mit Aktivitätsveränderungen in dieser Region am $\mathrm{Fuß}$ der dritten Stirnwindung. Informationstrukturelle Variationen führen hingegen zu Aktivierungen im pars triangularis (Bornkessel-Schlesewsky et al. 2010) und generelle Aspekte der Einfachheit der Informationsverarbeitung im bereits oben genannten Sulcus temporalis superior (Grewe et al 2007). Abbildung 6 illustriert die hier genannten neuroanatomischen Regionen. 


\section{Argumentverarbeitung aus einer sprachübergreifenden Perspektive}

Basierend auf den Erfolgen der generativen Grammatik im 20. Jahrhundert, ist es nicht verwunderlich, dass auch die in dieser Zeit entstehende Psycho- und Neurolinguistik sich an diesem theoretischen Ansatz orientierte und selbst Vertreter der Psycholinguistik, die die Grundannahmen der „Kompetenz-Performanz-Distinktion“ (Chomsky/Miller 1963), „Modularität" (Fodor 1983) oder „Serialität“ (Frazier/Fodor 1978) ablehnten, wesentliche Konsequenzen der Theorie - wenn auch möglicherweise unbewusst - mittrugen. So findet sich beispielsweise das Konzept der syntaktischen Strukturierung im Sinne der Annahmen Chomskys auch bei Vertretern interaktiver Ansätze (MacDonald et al. 1994). Wesentlich bedeutsamer für die Frage nach der Natur der Argumentinterpretation und ihren zugrunde liegenden Mechanismen war und ist die der Sprachwissenschaft der zweiten Hälfte des 20. Jahrhunderts innewohnende Überzeugung, dass man aus Beobachtungen zum Verarbeitungsverhalten im Englischen auf universelle Eigenschaften der Sprachverstehensarchitektur schließen könnte. Das hat zwei Konsequenzen: Einerseits bestimmte die frühe Verfügbarkeit des Verbs die Theoriebildung im Kontext der Argument-Verb-Relationen. Hier - wie zu Beginn des Aufsatzes bereits kurz erwähnt - gingen einzelne Arbeiten so weit anzunehmen, dass in verbfinalen Sprachen die Argumente bis zum Auftreten des Verbs entweder nur unverarbeitet gespeichert werden (Pritchett 1988, 1992) oder eine Art Vorverarbeitung stattfindet, bei der wesentliche Eigenschaften unterspezifiziert bleiben (Townsend/Bever 2001). Andererseits führte die Dominanz der Wortstellung für die inkrementelle Interpretation der Argumente zu einer Überbetonung dieser Eigenschaft als primärem Verarbeitungskonzept. Wortstellung, d.h. die lineare Position der Argumente zueinander, ist für das Englische mit Sicherheit bedeutsam (MacWhinney/Bates/Kliegl 1984), ${ }^{10}$ da es in der Regel die unmittelbare Identifizierung der grammatischen Funktion gestattet. Aus einer sprachübergreifenden Perspektive stellt die Dominanz der Wortstellung eine Ausnahme dar. Hier kommen Faktoren wie Belebtheit (Fore: Scott 1978), thematische Rolle (Belhare: Bickel 2006) oder Topikalität (Chinesisch: Li/Thompson 1981; Bisang 2006) eine deutlich bestimmendere Rolle bei der Argumentinterpretation zu.

Welche Anforderungen bestehen aber an eine Sprachverstehensarchitektur, wenn diese über die Einzelsprache hinaus Generalisierungen zur inkrementellen Argumentinterpretation erlauben soll? Verbfinalität und freie

10 Englisch ist gegenwärtig die einzige Sprache, die auf einem initialen unbelebten Argument einen lokalen Verarbeitungsnachteil im EEG in Form einer N400 zeigt (Weckerly/Kutas 1999). Hier ist tatsächlich die strukturelle Position, in dem ein Argument steht, bestimmend für dessen Interpretation und Handlungsprototypikalität. 
Wortstellung sind sicher wichtige Faktoren, die hier bereits genannt und besprochen wurden. Hinzu kommen aber noch Faktoren, die bisher keine Beachtung gefunden haben. Viele Sprachen der Welt erlauben die Auslassung von Argumenten (pro-drop Sprachen) und machen von dieser Möglichkeit auch regen Gebrauch (z.B. wird im Japanischen oder Türkischen zu 70\% das Subjekt in Sätzen mit transitiven Verben weggelassen). Andere Sprachen (z.B. Chinesisch) haben die Tendenz, als erstes Argument ein Topik zu präsentieren und dabei der grammatischen Funktion eher nachgeordnete Bedeutung zuzuordnen. Und was machen wir in Sprachen mit einem Ergativ-Absolutiv-Kasussystem, wenn wir ein Argument im Absolutiv verarbeiten?

Hier erweisen sich nun die in Abschnitt 3 anhand deutscher Beispiele eingeführten Interpretationsstrategien als ausgesprochen hilfreich. In den bisher untersuchten Sprachen scheint es demnach unabhängig von der Sprachfamilie so zu sein, dass das erste Argument, falls es keine Gegenevidenz gibt (z.B. Verbinformation oder Kasus), als einziges Argument einer intransitiven Relation interpretiert wird (Japanisch: Wolff et al. 2008; Türkisch: Demiral et al. 2008; Chinesisch: Wang et al. 2009; Hindi: Choudhary 2010). Diese Intransitivitätsannahme wird weder in pro-drop Sprachen (z.B. Türkisch: Demiral et al. 2008) noch in Topik-prominenten Sprachen (Chinesisch: Wang et al. 2009) durch Merkmale moduliert, die den Handlungsverursacher (Actor) in seinen prototypischen Eigenschaften absenken (z.B. Belebtheit). Eine notwendige Erweiterung zu einer transitiven Relation ist ebenso wie im Deutschen kostenträchtig (Japanisch: Wolff et al. 2008). Sollte während dieser Erweiterung deutlich werden, dass das erste Argument nicht Subjekt, d.h. das kongruierende Argument, oder Actor, der thematisch höchste Handlungsteilnehmer, ist, dann treten erhöhte Aktivierungen im neurophysiologischen Signal auf, die auf eine notwendig gewordene Reanalyse hindeuten (siehe Abbildung 4).

Der Versuch, einen Handlungsverursacher auch in Abwesenheit eines Verbs so schnell wie möglich zu identifizieren, scheint dabei die treibende Kraft zu sein. Insofern ist es auch nicht erstaunlich, dass - falls er durch ein anderes Element in seiner Existenz vorhergesagt wird - eine Verletzung von prototypischen Eigenschaften stets mit messbaren Aktivierungsveränderungen einhergeht.

Auch hier haben Wortstellung, Weglassbarkeit von Argumenten und Kasussystem keinen modulierenden Einfluss, wie man an den Beispielen in Abbildung 5 sehen kann.

Dass es dabei aber nicht nur um eine reine Bewertung der Güte eines Handlungsverursachers geht, sondern der Wettbewerb um diese Rolle das Verarbeitungsverhalten bestimmt, zeigen zwei weitere Beobachtungen aus dem Chinesischen (Philipp et al. 2008) und dem Tamil (Muralikrishnan et al. 2008). Während im Chinesischen und im Tamil, wie man in Abbildung 5 
Deutsch

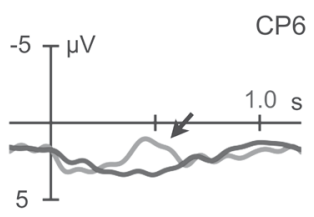

Chinesisch

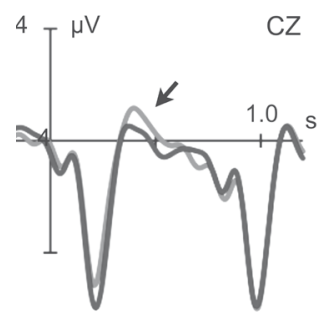

Deutsch

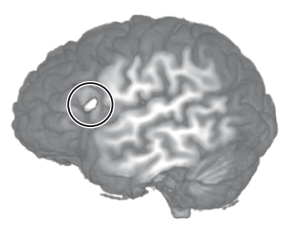

Hindi

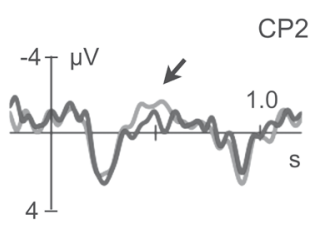

Türkisch

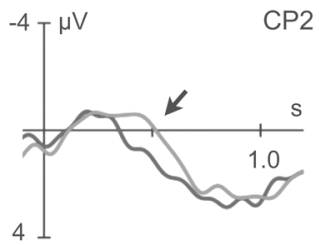

Japanisch

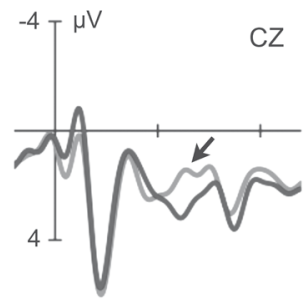

Abb. 4: Korrektur einer initialen Präferenz für ein handlungsverursachendes Actor-Argument (Deutsch: Bornkessel et al. 2005; Haupt et al. 2008; Türkisch: Bornkessel-Schlesewsky et al. (einger.), siehe ebenfalls Demiral et al. 2008; Chinesisch: Wang et al. 2009; Hindi: Choudhary et al. 2010; Japanisch: Wolff et al. 2007). Die EEGs stellen die Aktivitätsveränderungen am disambiguierenden Verb dar (Elektrodenpositionen sind in Abbildung 6 illustriert). Helle Linie: nicht-Actor-erst-Struktur; Dunkle Linie: Actor-erst-Struktur. Man beachte, dass die Kurven niemals absolut, d.h. im Koordinatensystem interpretiert werden, sondern nur relativ zueinander (siehe Fußnote 4). Die neuroanatomische Darstellung der korrespondierenden Aktivierungsmodulation im Deutschen zeigt den Pars opercularis (siehe Abbildung 6).

sehen kann, eine Negativierung im N400-Zeitfenster zu beobachten ist, wenn der Actor die Erwartungen an seine Eigenschaften (hier Belebtheit) nicht erfüllt, verändert sich in beiden Sprachen das neurophysiologische Aktivierungsmuster im Kontext von zwei unbelebten Handlungsteilnehmern qualitativ. Im Chinesischen zeigt sich nun nicht mehr eine N400, sondern eine Positivierung und im Tamil verändert sich die Latenz der Komponente, d.h. sie tritt nun signifikant früher auf. Wäre es eine reine Bewertung der argumentinhärenten Eigenschaften, so würde man nur eine Veränderung der Stärke der mit dieser verbundenen Komponente rechnen (Veränderung der Größe der N400). Das Auftreten eines anderen Musters, welches - und das muss man sich noch einmal kurz verdeutlichen - nur entstehen kann, wenn andere neuronale Netze miteinander interagieren, deutet darauf hin, dass der Wettbewerb von zwei unbelebten Argumenten 

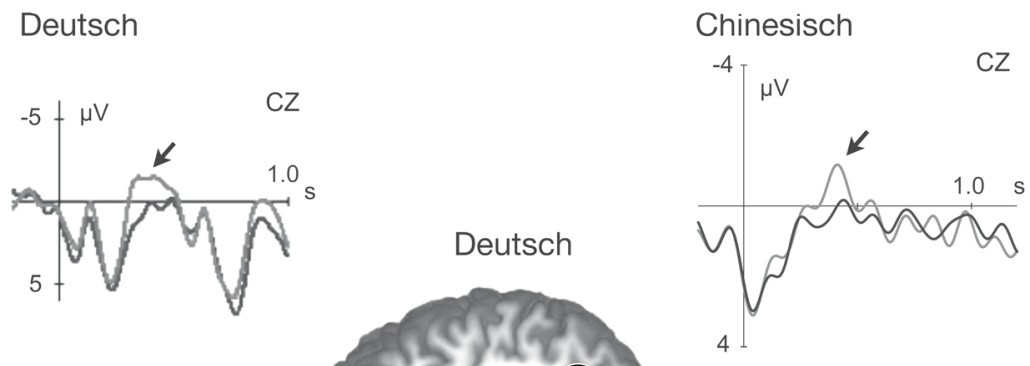

Hindi

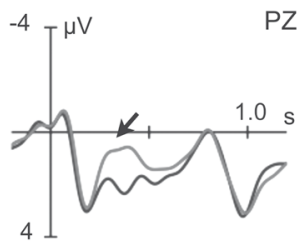

Chinesisch

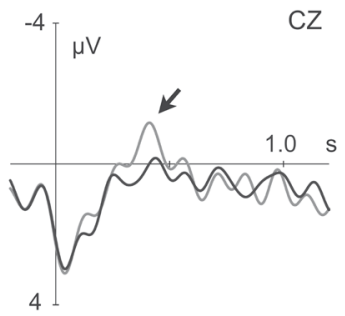

Tamil

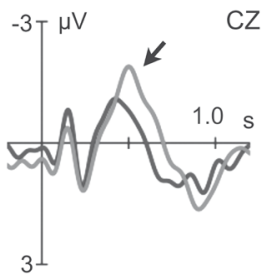

Abb. 5: Korrektur einer Erwartung für einen prototypischen Actor (Deutsch: Roehm et al. 2004, siehe ebenfalls Frisch/Schlesewsky 2001; Chinesisch: Philipp et al. 2008; Hindi: Schlesewsky et al. (ersch.); Tamil: Muralikrishnan et al. 2008). Alle Strukturen folgen dem gleichen Muster: Eine initiale NP signalisiert eine später folgende NP, die Handlungsverursacher ist. Ist die zweite NP unbelebt, erfolgt eine messbare Korrektur der Erwartung. Die Elektrodenpositionen sind in Abbildung 6 illustriert. Helle Linie: unbelebter Handlungsverursacher; Dunkle Linie: belebter Handlungsverursacher. Man beachte, dass die Kurven niemals absolut, d.h. im Koordinatensystem interpretiert werden, sondern nur relativ zueinander (siehe Fußnote 4). Die neuroanatomische Darstellung der korrespondierenden Aktivierungmodulation im Deutschen zeigt den Sulcus temporalis superior (siehe Abbildung 6).

eine völlig andere Qualität besitzt als die von zwei belebten Handlungsteilnehmern. Während im zweiten Fall der Actor prototypische Eigenschaften besitzt, kann dies im ersten Fall durch die Unbelebtheit beider Argumente zu keinem Zeitpunkt der Fall sein. Das führt - so deuten die Muster an zu alternativen Wettbewerbsstrategien. Wie sich Letzteres genau modellieren lässt, ist momentan noch nicht klar und bedarf weiterer Untersuchungen. Bringt man alle Beobachtungen zusammen, so ist das zentrale Konzept hinter allen bisherigen Daten offenkundig der Versuch, einem Argument sofort eine Actor-Rolle zuzuweisen und - sollte mehr als ein Argument vorhanden sein - diese hinsichtlich der Zuweisung dieser Rolle klar voneinander zu trennen. Alle Abweichungen führen zu erhöhter Verarbeitungskomplexität (z.B. durch Interferenz) und damit zu höheren Kosten. 
Doch hat die klare Identifizierung eines Actors und die Vermeidung von Interferenzen zwischen Argumenten nicht nur Vorteile hinsichtlich der Einfachheit der Informationsverarbeitung aus der Perspektive der Argument-Argument-Relation und der Integration dieser in den Verbrahmen. Wie Daten aus dem Türkischen zeigen (Demiral et al. (unter Begutachtung); Bornkessel-Schlesewsky/Schlesewsky 2008a) resultiert aus den Merkmalen und Eigenschaften des Arguments, d.h. den overten Kasusmerkmalen und prototypischen Actoreigenschaften, und der Position eines Argumentes in der linearen Sequenz eine Vorhersage für einen bestimmten Verbtyp. Die Abfolge Nominativ vor Akkusativ (und damit Actor vor Undergoer) erzeugt im Türkischen eine Erwartung für ein satzfinales Aktivverb. Erscheint an dessen Stelle ein Experiencerverb, so zeigen sich wieder einmal erhöhte Aktivierungen in Form einer N400, die wiederum als Kosten einer Erwartungsverletzung an der Syntax-Semantik-Schnittstelle interpretiert werden können. Invertiert man die Abfolge der Handlungsteilnehmer, so verändert sich auch die Erwartung des Verbtyps und man beobachtet nun eine N400 für das Aktivverb. Die Möglichkeit, aus der präverbalen Argumentrelation generalisierte Verbtypenschemata vorherzusagen, hat den klaren Vorteil einer schnelleren Integration der Information und damit einer effizienteren Kommunikation in Echtzeit.

\section{Argumentidentifizierung}

In den bisherigen Ausführungen haben wir stets vorausgesetzt, dass das menschliche Sprachverstehenssystem bei der Verarbeitung von Informationen in Echtzeit unmittelbar erkennt, wann ein lautliches Segment als Argument verarbeitet werden kann. Wir haben dabei implizit vorausgesetzt, dass ein Argument auch ohne Probleme als solches erkannt wird. Diese Annahme kann man machen und sie scheint empirisch, folgt man Clifton/ Spear/Abney (1991), Friederici (2002) und Bornkessel/Schlesewsky (2006a), auch richtig. Sie ist aber keineswegs trivial. Zwei Präliminarien sind dazu nämlich unabdingbar. Um etwas als ein Argument zu interpretieren, muss man diesem zuerst die potenzielle Fähigkeit für ein solches zuschreiben, d.h. es muss inhärente Merkmale besitzen, die nominal interpretierbar sind, und man muss es darüber hinaus auch noch als ein solches interpretieren können, d.h. es muss in einer potenziell nominal verträglichen Umgebung stehen. Der Einfachheit halber beschränken wir unsere folgenden Betrachtungen auf nominale Argumente und lassen Präpositionalphrasen, für die natürlich Gleiches gilt, hier unbeachtet. Erkennen wir innerhalb eines Textes bzw. eines Lautstroms eine segmentierbare und damit wahrscheinlich wortfähige Einheit, dann muss man diese in einem ersten Schritt hinsichtlich ihrer Wortkategorie klassifizieren. Das scheint notwendig und unabding- 
bar, da z.B. sowohl Nomen als auch Verben das Merkmal [+belebt] besitzen können, dieses aber in beiden Fällen unterschiedliche Konsequenzen besitzt. ${ }^{11}$ Entscheidend für die Zuweisung eines Wortkategoriemerkmals scheint, wie die experimentellen Daten suggerieren, die Erwartung zu sein, die aus den bisher verarbeiteten Informationen resultiert, bzw. wenn keine Erwartung vorhanden ist, die Position, in der das vermeintliche Wort auftritt. Dabei scheint Letzteres, wie wir zum Englischen argumentiert haben, nur eine Ausnahme zu sein, da wir ja nur in Sprachen mit freier Wortstellung in der ersten Position auf diese allein vertrauen müssen. Ansonsten weist uns die vorherige sprachliche Information den Weg. Das wohl bekannteste Experiment zu der Frage der Zuweisung von Wortkategorieinformation stammt aus der Arbeitsgruppe von Angela Friederici und beinhaltet zwei Arten von unerwarteten Weiterführungen einer Sequenz (Hahne/ Friederici 1999). In ungrammatischen Sätzen wie Das Hubn wurde im gehalten beobachteten die Autoren eine bei ca. 120 Millisekunden beginnende Negativierung, die damit nicht nur früher als die hier bisher berichtete N400 auftrat, sondern auch eine andere Verteilung auf der Kopfoberfläche zeigte. Diese Komponente (Early Left Anterior Negativity - ELAN ${ }^{12}$ ) steht nun in einem direkten Zusammenhang mit der Wortkategorieverarbeitung. Man

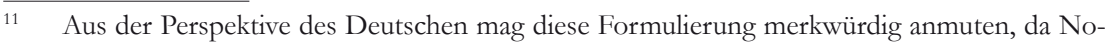
men und Verben ja i.d.R. eindeutig voneinander dissoziierbar sind (und das nicht nur allein durch die orthografische Konvention). Aus einer typologischen Sicht ist es aber keineswegs trivial, da Transkategorialität nicht nur allein Gegenstand deskriptiver Beschreibungen ist, wie man sie aus dem Chinesischen kennt (Bisang 2008), sondern generell ein Problem der linguistischen Theoriebildung darstellt. Hale/Marantz (1993) und andere Autoren haben im Kontext der Distributed Morphology (z.B. Harley/Noyer 1999) dieses Problem der Transkategorialität versucht zu modellieren. Danach besitzt ein lexikalischer Eintrag nur interpretierbare/formale Merkmale aber keine Wortkategorie. Letztere bekommt das Element nur im Kontext der Satzumgebung. So ist das Merkmal [+belebt] einem Item inhärent, wird aber durch die Umgebung entweder nominal charakterisierend oder subkategorisierend verstanden. Auch aus einer Verarbeitungsperspektive ist diese Idee attraktiv, da sie die initiale Verarbeitungsphase in Modellen wie Friederici (2002) oder Bornkessel/Schlesewsky (2006a) motiviert.

12 Die ELAN, eine früh auftretende Komponente mit topografischem Fokus auf der linken vorderen Schädeloberfläche, ist eigentlich eine Glaubenskomponente, d.h. die empirische Validierbarkeit ist so beschränkt, dass sie nur in derart ausgesuchten Kontexten erscheint, dass sie sich eigentlich jeder systematischen Untersuchung entzieht. Erstmals veröffentlicht wurde sie in einem Aufsatz von Neville et al. (1991). Die Studie berichtete eine frühe Negativierung zwischen 100 und 200 ms nach Stimulusonset, wenn die lokale Erwartung für eine Wortkategorie verletzt wird (*What did the scientist criticize Max's proof of?). In mehreren Studien zum Deutschen wurde diese Komponente in ähnlicher experimenteller Umgebung repliziert (Hahne/Friederici 1999). Kritik gab es aber stets aus zwei Richtungen. Während die eine argumentierte, dass der Satz potenziell weitergeführt werden kann (Die Gans wurde im gefüttert vorgefübrtem Zustand ausgestellt), kritisierte die andere, dass die Umgebung des Vergleichs, d.h. der prästimulus Bereich, keine seriöse physiologisch-empirische Auswertung erlaubt. Trotz aller Probleme zeigt die sprachübergreifende Transkategorialität (siehe Fußnote 11), dass es - wenn auch nicht diese Komponente - so doch einen frühen Schritt der Wortkategorisierung innerhalb des Sprachverstehens geben muss. 
kann argumentieren, dass die Komponente die Erwartungsverletzung widerspiegelt, die dann eintritt, wenn nach dem Präpositions-DeterminerKomplex das erwartete Nomen (z.B. Stall) nicht auftritt, sondern ein Wort verarbeitet werden muss, welches verbale Eigenschaften (Partizip) besitzt. Doch während die absolute Zeit der Komponenten zueinander nur einen ersten Hinweis auf einen frühen Prozess der Wortkategorieidentifizierung gibt, zeigte eine weitere experimentelle Manipulation die wahre Bedeutung des dort ablaufenden Prozesses. Friederici und Kollegen (Hahne/Friederici 2002) untersuchten darüber hinaus ungrammatische Sätze, die nicht nur eine Wortkategorieverletzung enthielten, sondern bei denen das Partizip, wie in Das Hubn wurde im gebügelt, auch noch einen Verstoß gegen unser Weltwissen enthielt, denn wir besitzen kein Alltagsszenario, in dem gebügelte Hühner vorkommen. Betrachtet man beide Verletzungen separat, dann zeigen sich - wie oben bereits erwähnt - zeitlich und räumlich (topographisch) unterschiedliche Effekte. Aus der Annahme, dass Sprachverstehensprozesse interagieren und/oder zeitgleich alle Informationsarten verarbeiten, leitete sich die Hypothese ab, dass in der gekoppelten Verletzungsbedingung beide Komponenten zu beobachten sein sollten. Doch das war genau nicht der Fall: Friederici und Kollegen beobachteten bei diesen Sätzen exakt das gleiche neurophysiologische Aktivierungsmuster wie bei einer einfachen Wortkategorieverletzung. Daraus kann man nicht nur schließen, dass der Prozess der Wortkategorie anderen Sprachverstehensprozessen vorangeht, sondern dass er auch in der Lage zu sein scheint, spätere Prozesse zu blockieren, d.h. nach Erkennung der Verletzung die Verarbeitung abzubrechen. Diese Eigenschaft der Blockierung und der Einzigartigkeit dieser im Kontext der Wortkategorieerkennung wurde für das Niederländische durch van den Brink und Hagoort (2004) bestätigt (für eine ausführliche Diskussion siehe Bornkessel-Schlesewsky/Schlesewsky 2009b).

Hat man also einen nominalen Ausdruck als einen solchen identifiziert, so gibt es nun wiederum zwei Möglichkeiten zur Interpretation. Auch dazu wieder ein Beispiel. Bei der inkrementellen Verarbeitung des sich aufbauenden Satzes Vielleicht hatte der Vater der Sängerin ... muss man im Moment des Erscheinens der ambigen Nominalphrase „entscheiden“, ob man diese als Dativ und damit als Argument eines möglicherweise folgenden Verbs interpretiert, oder ob man sie als Genitiv interpretiert und damit zum Adjunkt und Modifizierer des vorangehenden Nomens macht. Für den ersten Fall würde man erwarten, dass mit dem Auftreten eines intransitiven Verbs (geschlafen) eine neurophysiologische Aktivierungsveränderung zu beobachten sein sollte. Wäre die Entscheidung hingegen für den postnominalen Genitiv gefallen, dann sollte ein transitives Verb (folgen) aktivierungsverändernd sein. Bereits in früheren psycholinguistischen Studien (Abney 1989; Clifton/Spear/Abney 1991; Boland/Boehm-Jernigan 1998; Schütze/Gibson 1999) beobachtet man hier ein relativ klares Muster (siehe Augurzky/Schle- 
sewsky 2011). Auch wenn die durch Vater induzierte mögliche Verwandtschaftsrelation der Dativlesart entgegen wirkt, gibt es trotzdem eine klare Tendenz, die ambige NP als Argument und nicht als Adjunkt zu interpretieren. Gleiches gilt übrigens auch für Präpositionalphrasen, die, wie Juranek (2005) mit Hilfe von EEG-Messungen zeigen konnte, ebenfalls einer Argument-über-Adjunkt-Präferenz folgen.

Zusammenfassend kann man also aus einer Sprachverstehensperspektive argumentieren, dass bedeutungstragende Einheiten, die beispielsweise aus einem Lautstrom segmentiert und als nicht-verbfähige Information eingeordnet werden, mit hoher Wahrscheinlichkeit als Argument interpretiert werden. Diese Vorgehensweise hat dabei sowohl den Vorteil, dass Ereignisse im verbnahen engeren Sinne (d.h. wie zum Beispiel über lexikalische Dekomposition beschreibbar) schnell und effizient aufgebaut werden können, als auch die Konsequenz des Aufbaus maximal einfacher Strukturen. ${ }^{13}$ Auf diesen ersten Schritten bauen dann die in den Abschnitten 2-4 geschilderten relationalen und dependenzbildenden Prozesse auf.

\section{Ausblick}

Auch wenn die hier präsentierte Datenlage relativ stabil und klar erscheint und die vorgeschlagenen Annahmen stützt, bleiben natürlich doch noch einige offene Fragen. Die wohl einfachste betrifft die weitere Validierung mit Hilfe von bisher nicht untersuchten Sprachen. Dazu gehört mit Sicherheit die Hinzunahme weiterer Ergativsprachen (z.B. Baskisch) aber auch die Hinwendung zu verbinitialen Sprachen (z.B. Irisch). Auch gilt es natürlich zu untersuchen, wie die Interaktion der Prominenzskalen entsteht und wie bzw. ob überhaupt die in Experimenten zu beobachtende Hierarchisierung zwischen diesen Skalen Widerspiegelung in absoluten Häufigkeiten und Übergangswahrscheinlichkeiten findet. Der aber vielleicht wichtigste Schritt für ein umfassenderes Verständnis der dynamischen Interpretation liegt in der Untersuchung von Argumentrelationen, bei denen eine oder mehrere

13 Unter der hier vertretenen Annahme der maximalen Einfachheit (Bornkessel/Schlesewsky 2006; Bornkessel-Schlesewsky/Schlesewsky 2009c) werden andere Wortkategorien nur dann angenommen, wenn explizite Information für diese vorliegt. In Abwesenheit dieser werden alle segmentierbaren Einheiten entweder als Verb oder als nominales Argument präferiert interpretiert. Das Prinzip der Einfachheit („Minimality principle: In the absence of explicit information to the contrary, the human language comprehension system assigns minimal structures. This entails that only required dependencies and relations are created“, Bornkessel/Schlesewsky 2006, S. 790), welches in seiner Philosophie dem „minimal everything principle“ (Fodor 1998; Inoue/Fodor 1995) ähnelt, appliziert auf allen Ebenen des Sprachverstehens. Im Kontext unseres eigenen Modells (Bornkessel 2002; Schlesewsky/Bornkessel 2004; Bornkessel/Schlesewsky 2006a; Bornkessel-Schlesewsky/Schlesewsky 2008b) fokussieren wir insbesondere auf die syntaktische, semantische Dimension und auf das Interface zwischen beiden. 
Argumente Quantoren enthalten. Hier besteht derzeit sowohl Forschungsals auch Modellierungsbedarf. Interessant ist der Punkt deshalb, da beispielsweise bei doppelt quantifizierenden Strukturen zwar theoretisch mehrere Lesarten bestehen, in der Echtzeitkommunikation diese Ambiguitäten aber nicht vorzukommen scheinen. Darüber hinaus sind, wenn man diese Strukturen in Reaktionszeitexperimenten (z.B. Bott/Schlotterbeck 2010) untersucht, die Reaktionszeiten mit einem Mittel von über 2 Sekunden in einem Bereich, der jenseits dessen ist, was man von Studien in anderen Domänen der Argumentverarbeitung kennt. Hier werden i.d.R. 1000 ms deutlich unterschritten. Diese Beobachtung deutet darauf hin, dass derartige Strukturen eher mit Hilfe allgemeinkognitiver Problemlösungsstrategien gelöst werden, als mit denjenigen Strategien, die wir in der Echtzeitkommunikation finden. $\mathrm{Da}$ es sich bei diesen aber nichtsdestoweniger um sprachbasierte Phänomene handelt, die die Relation der Argumente zum Teil mitbestimmen, verlangen diese Eigenschaften daher auch eine Integration in den in diesem Aufsatz vorgestellten Rahmen.

Offen bleibt natürlich, in welcher Relation die hier vorgestellten Daten zu existierenden Grammatiktheorien stehen, die ja alle keine zeitliche Dimension besitzen. Ob diese, wenn man Daten der Art, wie sie hier vorgestellt wurden, wirklich ernst nimmt, mit dieser Dimension umgehen können, oder ob es einer völlig neuen Art der grammatischen Theoriebildung bedarf, wird die Zukunft zeigen.

\section{Anhang}
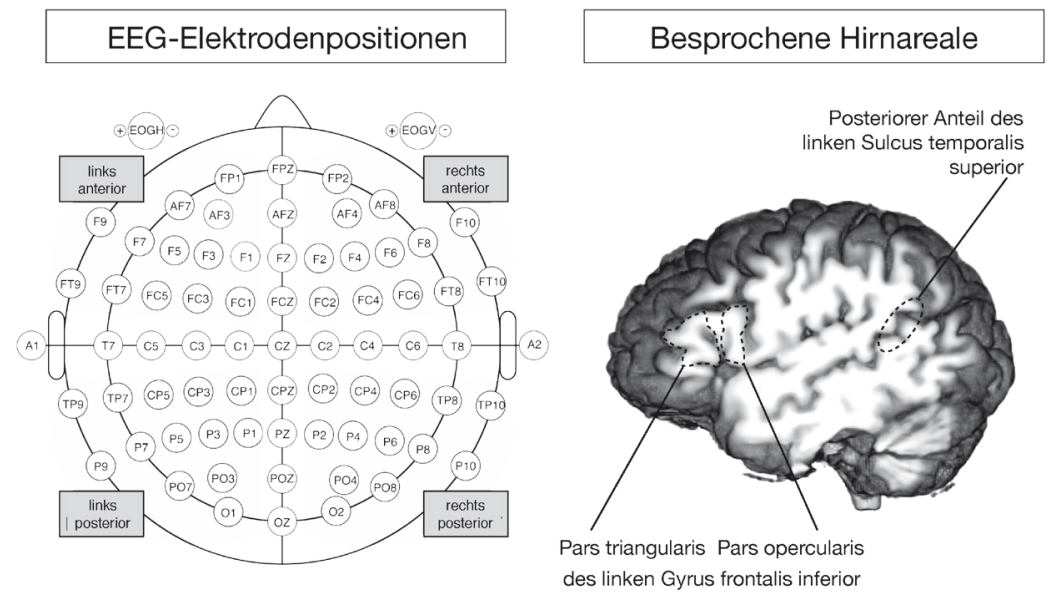

Abb. 6 
Abbildung 6 zeigt auf der linken Seite die Elektrodenpositionen, die standardmäßig in EEG-Experimenten verwendet werden (adaptiert aus Bornkessel-Schlesewsky/Schlesewsky 2009a). Sie geben auch die in den anderen Abbildungen genannten Elektrodenpositionen wieder. Auf der rechten Seite werden die ungefähren neuroanatomischen Positionen genannt und bezeichnet, die im vorliegenden Aufsatz erwähnt werden.

\section{Literatur}

Abney, Steven (1989): A computational model of human parsing. In: Journal of Psycholinguistic Research 18, S. 129-144.

Aissen, Judith (2003): Differential object marking: iconicity vs. economy. In: Natural Language and Linguistic Theory 21, S. 435-483.

Augurzky, Petra/Schlesewsky, Matthias (2011): Prosodic phrasing and transitivity in head-final sentence comprehension: ERP evidence from German ambiguous DPs. In: Yamashita, Hiroko et al. (Hg.): Processing and producing head-final structures. (= Studies in Theoretical Psycholinguistics 38). Berlin, S. 69-91.

Berger, Hans (1929): Über das Elrektrenkephalogramm des Menschen. In: Archiv für Psychiatrie und Nervenkrankheiten 87, S. 527-570.

Bergmann, Christopher (2010): Konstruktionen oder Strukturen? Zum Einfluss der Wortfrequenz auf die Sprachverarbeitung von Subjekt/Objekt-Ambiguitäten im Deutschen. Unveröff. Magisterarbeit. Mainz.

Bickel, Balthasar (2006): Clause-level vs. predicate-level linking. In: Bornkessel et al. (Hg.), S. 155-190.

Bisang, Walter (2006): From meaning to syntax - semantic roles and beyond. In: Bornkessel et al. (Hg.), S. 191-236.

Bisang, Walter (2008): Precategoriality and syntax-based parts of speech: the case of Late Archaic Chinese. In: Studies in Language 32, S. 568-589.

Boland, Julie/Boehm-Jernigan, Heather (1998): Lexical constraints and prepositional phrase attachment. In: Journal of Memory and Language 39, S. 684-719.

Bornkessel, Ina (2002): The Argument Dependency Model: a neurocognitive approach to incremental interpretation. (= MPI Series in Cognitive Neuroscience 28). Leipzig.

Bornkessel, Ina/Schlesewsky, Matthias (2006a): The Extended Argument Dependency Model: a neurocognitive approach to sentence comprehension across languages. In: Psychological Review 113, S. 787-821.

Bornkessel, Ina/Schlesewsky, Matthias (2006b): Generalised semantic roles and syntactic templates: a new framework for language comprehension. In: Bornkessel et al. (Hg.), S. 327-353.

Bornkessel, Ina/Fiebach, Christian J./Friederici, Angela D. (2004): On the cost of syntactic ambiguity in human language comprehension: an individual differences approach. In: Cognitive Brain Research 21, S. 11-21. 
Bornkessel, Ina/Schlesewsky, Matthias/Friederici, Angela D. (2003): Eliciting thematic reanalysis effects: the role of syntax-independent information during parsing. In: Language and Cognitive Processes 18, S. 268-298.

Bornkessel, Ina et al. (2004): Multi-dimensional contributions to garden path strength: dissociating phrase structure from case marking. In: Journal of Memory and Language 51, S. 495-522.

Bornkessel, Ina et al. (2005): Who did what to whom? The neural basis of argument hierarchies during language comprehension. In: NeuroImage 26, S. 221-233.

Bornkessel, Ina et al. (Hg.) (2006): Semantic role universals and argument linking: theoretical, typological, and psycholinguistic perspectives. (= Trends in Linguistics, Studies and Monographs 165). Berlin/New York.

Bornkessel-Schlesewsky, Ina/Schlesewsky, Matthias (2008a): Unmarked transitivity: a processing constraint on linking. In: van Valin, Robert D. (Hg.): Investigations of the syntax-semantics-pragmatics interface. (= Studies in Language Companion Series 105). Amsterdam u.a., S. 413-434.

Bornkessel-Schlesewsky, Ina/Schlesewsky, Matthias (2008b): An alternative perspective on „semantic P600“ effects in language comprehension. In: Brain Research Reviews 59, S. 55-73.

Bornkessel-Schlesewsky, Ina/Schlesewsky, Matthias (2009a): Processing syntax and morphology: a neurocognitive perspective. (= Oxford Surveys in Syntax and Morphology 6). Oxford u.a.

Bornkessel-Schlesewsky, Ina/Schlesewsky, Matthias (2009b): The role of prominence information in the real time comprehension of transitive constructions: a crosslinguistic approach. In: Language and Linguistics Compass 3, S. 19-58.

Bornkessel-Schlesewsky, Ina/Schlesewsky, Matthias (2009c): Minimality as vacuous distinctness: evidence from cross-linguistic sentence comprehension. In: Lingua 119, S. 1541-1559.

Bornkessel-Schlesewsky, Ina/Grewe, Tanja/Schlesewsky, Matthias (2010): Prominence vs. aboutness in sequencing: a functional distinction within the left inferior frontal gyrus. In: Brain and Language. [doi: 10.1016/j.bandl.2010.06.004].

Bornkessel-Schlesewsky, Ina/Schlesewsky, Matthias/von Cramon, D. Yves (2009): Word order and Broca's region: evidence for a supra-syntactic perspective. In: Brain and Language 111, S. 125-139.

Bornkessel-Schlesewsky, Ina et al. (eingereicht): Think globally: cross-linguistic variation in electrophysiological activity during sentence comprehension. In: Brain and Language.

Bott, Oliver/Schlotterbeck, Fabian (2010): An investigation into the computational complexity of quantifying expressions. Paperpräsentation bei der 23rd Annual Meeting of the CUNY Conference on Human Sentence Processing, New York 18.-21. März 2010.

Chomsky, Noam/Miller, George A. (1963): Introduction to the formal analysis of natural languages. In: Luce, R. Duncan/Bush, Robert R./Galanter, Eugene (Hg.): Handbook of mathematical psychology. New York u.a., S. 269-321. 
Choudhary, Kamal K. (2010): Incremental argument interpretation in a split ergative language: neurophysiological evidence from Hindi. Diss. Leipzig.

Choudhary, Kamal K. et al. (2009): The N400 as a correlate of interpretively-relevant linguistic rules: evidence from Hindi. In: Neuropsychologia 47, S. 3012-3022.

Choudhary, Kamal K. et al. (2010): An actor-preference in a split-ergative language: electrophysiological evidence from Hindi. Posterpräsentation bei der 23rd Annual CUNY Conference on Human Sentence Processing, New York 18.-21. März 2010.

Clifton Jr., Charles/Speer, Shari/Abney, Steven P. (1991): Parsing arguments: phrase structure and argument structure as determinants of initial parsing decisions. In: Journal of Memory and Language 30, S. 251-271.

Comrie, Bernard (1989): Linguistic universals and language typology: syntax and morphology. 2. Aufl. Oxford.

Crocker, Matthew W. (1994): On the nature of the principle-based sentence processor. In: Clifton Jr., Charles/Frazier, Lyn/Rayner, Keith (Hg.): Perspectives on sentence processing. Hillsdale, S. 245-266.

Croft, William (2003): Typology and universals. 2. Aufl. Cambridge u.a.

DeLancey, Scott (1981): An interpretation of split ergativity and related patterns. In: Language 57, S. 626-657.

Demiral, Şükrü Barış/Schlesewsky, Matthias/Bornkessel-Schlesewsky, Ina (2008): On the universality of language comprehension strategies: evidence from Turkish. In: Cognition 106, S. 484-500.

Demiral, Şükrü Barış/Schlesewsky, Matthias/Bornkessel-Schlesewsky, Ina (unter Begutachtung): Anticipating clause-final verbs: the interaction of prominence hierarchies in Turkish sentence comprehension. [Zur Veröffentlichung eingereichtes Manuskript].

de Swart, Peter (2007): Cross-linguistic variation in object marking. Diss. Utrecht.

Dowty, David (1991): Thematic proto-roles and argument selection. In: Language 67, S. 547-619.

Fanselow, Gisbert (2000): Optimal exceptions. In: Stiebels, Barbara/Wunderlich, Dieter (Hg.): Lexicon in Focus. (= Studia grammatica 45). Berlin, S. 173-209.

Farke, Hildegard (1994): Grammatik und Sprachverarbeitung. Zur Verarbeitung syntaktischer Ambiguitäten. Opladen.

Fodor, Janet D. (1998): Learning to parse. In: Journal of Psycholinguistic Research 27, S. 285-319.

Fodor, Jerry A. (1983): Modularity of mind. An essay on faculty psychology. Cambridge, MA.

Foley, William A./van Valin, Robert D. (1984): Functional syntax and universal grammar. (= Cambridge Studies in Linguistics 38). Cambridge u.a.

Frazier, Lyn/Fodor, Janet D. (1978): The sausage machine: a new two-stage parsing model. In: Cognition 6, S. 291-326. 
Frazier, Lyn/Flores d'Arcais, Giovanni B. (1989): Filler-driven parsing: a study of gap filling in Dutch. In: Journal of Memory and Language 28, S. 331-344.

Friederici, Angela D. (2002): Towards a neural basis of auditory sentence processing. In: Trends in Cognitive Sciences 6, S. 78-84.

Friederici, Angela D. et al. (2006): Processing linguistic complexity and grammaticality in the left frontal cortex. In: Cerebral Cortex 16, S. 1709-1717.

Frisch, Stefan/Schlesewsky, Matthias (2001): The N400 indicates problems of thematic hierarchizing. In: Neuroreport 12, S. 3391-3394.

Garnsey, Susan M. (1993): Event-related brain potentials in the study of language: an introduction. In: Language and Cognitive Processes 8, S. 337-356.

Gordon, Peter C./Hendrick, Randall/Johnson, Marcus (2001): Memory interference during language processing. In: Journal of Experimental Psychology: Learning, Memory and Cognition 27, S. 1411-1423.

Grewe, Tanja et al. (2005): The emergence of the unmarked: a new perspective on the language-specific function of Broca's area. In: Human Brain Mapping 26, S. 178190.

Grewe, Tanja et al. (2006): Linguistic prominence and Broca's area: the influence of animacy as a linearization principle. In: Neuroimage 32, S. 1395-1402.

Grewe, Tanja et al. (2007): The role of the posterior superior temporal sulcus in the processing of unmarked transitivity. In: Neuroimage 35, S. 343-352.

Grimshaw, Jane B. (1990): Argument structure. (= Linguistic Inquiry Monographs 18). Cambridge, MA u.a.

Hahne, Anja/Friederici, Angela D. (1999): Electrophysiological evidence for two steps in syntactic analysis: early automatic and late controlled processes. In: Journal of Cognitive Neuroscience 11, S. 194-205.

Hahne, Anja/Friederici, Angela D. (2002): Differential task effects on semantic and syntactic processes as revealed by ERPs. In: Cognitive Brain Research 13, S. 339-356.

Halle, Morris/Marantz, Alec (1993): Distributed Morphology and the pieces of inflection. In: Hale, Kenneth/Keyser, Samuel Jay (Hg.) The view from building 20. Essays in linguistics in honor of Sylvain Bromberger. (= Current Studies in Linguistics Series 24). Cambridge, MA, S. 111-176.

Harley, Heidi/Noyer, Rolf (1999): State-of-the-article: distributed morphology. In: Glot International 4, S. 3-9.

Haupt, Friederike S. et al. (2008): The status of subject-object reanalyses in the language comprehension architecture. In: Journal of Memory and Language 59, S. 54-96.

Hemforth, Barbara/Konieczny, Lars/Strube, Gerhard (1993): Incremental syntax processing and parsing strategies. In: Proceedings of the 15th Annual Conference of the Cognitive Science Society. Hillsdale, S. 539-545.

Hopper, Paul/Thompson, Sandra A. (1980): Transitivity in grammar and discourse. In: Language 56, S. 251-299. 
Inoue, Atsu/Fodor, Janet D. (1995): Information-paced parsing of Japanese. In: Mazuka, Reiko/Nagai, Noriko (Hg.): Japanese sentence processing. Hillsdale, S. 9-63.

Jackendoff, Ray (1972): Semantic interpretation in generative grammar. (= Studies in Linguistics Series 2). Cambridge, MA.

Janssen, Ulrike/Wiese, Richard/Schlesewsky, Matthias (2006): Electrophysiological responses to violations of morphosyntactic and prosodic features in derived German nouns. In: Journal of Neurolinguistics 19, S. 466-482.

Juranek, Katja (2005): Relationale Aspekte des Sprachverstehens: Die Verarbeitung von Argumenten und Adjunkten. Magisterarb. Leipzig.

Kolk, Herman H.J. et al. (2003): Structure and limited capacity in verbal working memory: a study with event-related potentials. In: Brain and Language 85, S. 1-36.

Konieczny, Lars et al. (1997): The role of lexical heads in parsing: evidence from German. In: Language and Cognitive Processes 12, S. 307-348.

Kuperberg, Gina R. et al. (2003): Electrophysiological distinctions in processing conceptual relationships within simple sentences. In: Cognitive Brain Research 17, S. $117-129$.

Kutas, Marta/Hillyard, Steven A. (1980): Reading senseless sentences: brain potentials reflect semantic incongruity. In: Science 207, S. 203-205.

Lenerz, Jürgen (1977): Zur Abfolge nominaler Satzglieder im Deutschen. (= Studien zur deutschen Grammatik 5). Tübingen.

Lewis, Richard L./Vasishth, Shravan (2005): An activation-based model of sentence processing as skilled memory retrieval. In: Cognitive Science 29, S. 1-45.

Li, Charles N./Thompson, Sandra A. (1981): Mandarin Chinese: a functional reference grammar. Berkeley u.a.

MacDonald, Maryellen C./Pearlmutter, Neal J./Seidenberg, Mark S. (1994): The lexical nature of syntactic ambiguity resolution. In: Psychological Review 101, S. 676703.

MacWhinney, Brian/Bates, Elizabeth/Kliegl, Reinhold (1984): Cue validity and sentence interpretation in English, German and Italian. In: Journal of Verbal Learning and Verbal Behavior 23, S. 127-150.

Marslen-Wilson, William (1973): Linguistic structure and speech shadowing at very short latencies. In: Nature 244, S. 522-533.

Müller, Gereon (1999): Optimality, markedness and word order in German. In: Linguistics 37, S. 777-818.

Muralikrishnan, R./Schlesewsky, Matthias/Bornkessel-Schlesewsky, Ina (2008): Universal and cross-linguistic influences on the processing of word order and animacy: neurophysiological evidence from Tamil. Posterpräsentation bei der 21st Annual CUNY Conference on Human Sentence Processing, Chapel Hill, NC.

Neville, Helen J. et al. (1991): Syntactically based sentence processing classes: evidence from event-related potentials. In: Journal of Cognitive Neuroscience 6, S. 233-255. 
Philipp, Markus et al. (2008): The role of animacy in the real time comprehension of Mandarin Chinese: evidence from auditory event-related brain potentials. In: Brain and Language 105, S. 112-133.

Primus, Beatrice (1999): Cases and thematic roles: ergative, accusative and active. (= Linguistische Arbeiten 393). Tübingen.

Pritchett, Bradley L. (1988): Garden path phenomena and the grammatical basis of language processing. In: Language 64, S. 539-576.

Pritchett, Bradley L. (1992): Grammatical competence and parsing performance. Chicago u.a.

Röder, Brigitte et al. (2002): Brain activation modulated by the comprehension of normal and pseudo-word sentences of different processing demands: a functional magnetic resonance imaging study. In: Neuroimage 15, S. 1003-1014.

Roehm, Dietmar et al. (2004): Fractionating language comprehension via frequency characteristics of the human EEG. In: Neuroreport 15, S. 409-412.

Roehm, Dietmar et al. (2007): To predict or not to predict: influences of task and strategy on the processing of semantic relations. In: Journal of Cognitive Neuroscience 19, S. $1259-1274$.

Schlesewsky, Matthias/Bornkessel, Ina (2003): Ungrammaticality detection and garden path strength: a commentary on Meng and Bader's (2000) evidence for serial parsing. In: Language and Cognitive Processes 18, S. 299-311.

Schlesewsky, Matthias/Bornkessel, Ina (2004): On incremental interpretation: degrees of meaning accessed during sentence comprehension. In: Lingua 114, S. $1213-$ 1234.

Schlesewsky, Matthias/Choudhary, Kamal K./Bornkessel-Schlesewsky, Ina (ersch.): Grammatical transitivity vs. interpretive distinctness: The case for a separation of two levels of representation that are often conflated. In: Brandt, Patrick/García García, Marco (Hg.): Transitivity. Form, meaning, acquisition, and processing. Amsterdam.

Schriefers, Herbert/Friederici, Angela D./Kühn, Katja (1995): The processing of locally ambiguous relative clauses in German. In: Journal of Memory and Language 34, S. $499-520$.

Schütze, Carson T./Gibson, Edward (1999): Argumenthood and English prepositional phrase attachment. In: Journal of Memory and Language 40, S. 409-431.

Scott, Graham (1978): The Fore language of Papua New Guinea. (= Pacific Linguistics, Series B, 47). Canberra.

Silverstein, Michael (1976): Hierarchy of features and ergativity. In: Dixon, Robert M. W. (Hg.): Grammatical categories in Australian languages. (= Linguistic Series 22). Canberra, S. 112-171.

Sturt, Patrick/Crocker, Matthew W. (1996): Monotonic syntactic processing: a crosslinguistic study of attachment and reanalysis. In: Language and Cognitive Processes 11, S. 449-494. 
Townsend, David J./Bever, Thomas G. (2001): Sentence comprehension: the integration of habits and rules. Cambridge, MA u.a.

Urbach, Thomas P./Kutas, Marta (2010): Quantifiers more or less quantify on-line: ERP evidence for partial incremental interpretation. In: Journal of Memory and Language 2, S. 158-179.

Uszkoreit, Hans (1986): Constraints on order. In: Linguistics 24, S. 883-906.

van den Brink, Daniëlle/Hagoort, Peter (2004): The influence of semantic and syntactic context constraints on lexical selection and integration in spoken-word comprehension as revealed by ERPs. In: Journal of Cognitive Neuroscience 16, S. 1068-1084.

van Dyke, Julie A./McElree, Brian (2006): Retrieval interference in sentence comprehension. In: Journal of Memory and Language 55, S. 157-166.

Vosse, Thea/Kempen, Gerard (2000): Syntactic assembly in human parsing: a computational model based on competitive inhibition and lexicalist grammar. In: Cognition 75 , S. 105-143.

Wang, Luming et al. (2009): Exploring the nature of the subject'-preference: evidence from the online comprehension of simple sentences in Mandarin Chinese. In: Language and Cognitive Processes 24, S. 1180-1226.

Weckerly, Jill/Kutas, Marta (1999): An electrophysiological analysis of animacy effects in the processing of object relative sentences. In: Psychophysiology 36, S. 559570.

Wolff, Susann/Schlesewsky, Matthias/Bornkessel-Schlesewsky, Ina (2007): The interaction of universal and language-specific properties in the neurocognition of language comprehension: evidence from the processing of word order permutations in Japanese. In: Journal of Cognitive Neuroscience (Supplement) 107, S. 133-157.

Wolff, Susann et al. (2008): The neural mechanisms of word order processing revisited: electrophysiological evidence from Japanese. In: Brain and Language 107, S. 133157.

Wunderlich, Dieter (1997): Cause and the structure of verbs. In: Linguistic Inquiry 28, S. 27-68. 
